\title{
Extranodal Natural Killer/T-Cell Lymphoma of the Nasal Type with Skin Metastases
}

\author{
Fadi Al Akhrass ${ }^{a}$ Brooklyn Hensley $^{c}$ Lillian Thomas $^{b}$ \\ Raymond Elsoueidi ${ }^{d}$ \\ Departments of ${ }^{a}$ Infectious Disease and ${ }^{b}$ Hematology/Oncology, Pikeville Medical Center, \\ and ${ }^{C}$ College of Osteopathic Medicine, University of Pikeville, Pikeville, Ky., and \\ ${ }^{\mathrm{d}}$ Appalachian Regional Healthcare Cancer Center, Hazard, Ky., USA
}

\section{Key Words}

Extranodal natural killer/T-cell lymphoma $\cdot$ Nasal type $\cdot$ Skin metastases

\begin{abstract}
Extranodal natural killer/T-cell lymphoma (ENKL) of the nasal type is a rare, clinically aggressive disease. ENKL of the nasal type is often localized in the upper aerodigestive tract, including the nasal cavity, nasopharynx, paranasal sinuses, tonsils, hypopharynx and larynx, and usually presents as stage I/II. Extranasal involvement can occur, and a common site of extranasal involvement or metastatic disease includes the skin. Identifying skin metastases is important for the appropriate staging and treatment. We report a case of ENKL of the nasal type that presented with localized disease and subsequent skin lesions that were consistent with skin metastases.

C) 2016 The Author(s)

Published by S. Karger AG, Basel
\end{abstract}

\section{Case Presentation}

A 55-year-old Caucasian female with a history of hypothyroidism and splenectomy presented with complaints of swelling and pain involving the nose and the left face, nasal stuffiness and blood-stained nasal discharge for 3 months. She reported a painful, white mass in the left nostril, which had progressively increased in size, as well as dysphagia to solid food and profound fatigue. Physical examination showed enlargement of the nose with bilaterally widened nasal cavities, more pronounced on the left side. She had a mass in the left nostril and a $2 \times 3-\mathrm{cm}$ ulcerated palatal lesion. MRI of the face with contrast medium revealed the

\section{KARGER}

Raymond Elsoueidi

Appalachian Regional Healthcare Cancer Center

110 Medical Center Drive

Hazard, KY 41701 (USA)

E-Mail elsoueidimd@yahoo.com 
Al Akhrass et al.: Extranodal Natural Killer/T-Cell Lymphoma of the Nasal Type with Skin Metastases

presence of an $8.3 \times 4.8-\mathrm{cm}$ heterogeneous mass lesion involving both nasal airways with obstruction and destruction of the nasal septum (fig. 1). There was extension into the underlying hard palate and into the left ethmoid, sphenoid, left maxillary sinuses, soft palate and uvula. Nasal-mass and oral-lesion biopsies were performed. Histological findings showed a dense, diffuse, pandermal proliferation of atypical lymphoid cells with eosinophilic cytoplasm, hyperchromatic nuclei, irregular nuclear membranes and inconspicuous nucleoli. Extensive apoptosis, prominent angiocentricity, focal epidermotropism and dermal necrosis were noted (fig. 2). The tumor cells were strongly immunoreactive for T-cell-restricted intracellular antigen (TIA-1), granzyme, CD8, CD2, CD56 and cytoplasmic CD3. A few cells were immunoreactive for CD5 and CD4. Epstein-Barr virus (EBV)-encoded RNA (EBER) in situ hybridization was positive in the majority of the neoplastic cells. The cells were negative for CD57. These findings were consistent with natural killer/T-cell lymphoma (NKL). Bone marrow biopsy was negative for lymphomatous involvement. PET scan revealed a large, hypermetabolic lytic lesion involving the facial bones, nasal cavity and left maxilla. The lingual tonsils were enlarged and mildly hypermetabolic. There was also a hypermetabolic $11 \times$ 18-mm mass on the left anteromedial pleural base, a polypoid mass in the gastric fundus. An esophagogastroduodenoscopy ruled out gastric involvement. She was diagnosed with stage II NKL. The patient was treated with radiation and systemic chemotherapy with the DICE regimen (dexamethasone/ifosfamide/cisplatin/etoposide) with $20 \%$ dose reduction. Later on, she was found to have multiple erythematous, violaceous, well-circumscribed, nodular lesions over the back and bilateral thighs (fig. 3). The $3 \times 3$-cm skin lesion on the left thigh was surgically excised, and the histopathological finding was consistent with the previous nasal biopsy. Further treatment was complicated by myocardial infarction that required a delay in treatment. As a consequence, there was subsequent disease progression, and the patient passed away 3 months after initiating treatment.

\section{Discussion}

Extranodal NKL (ENKL) of the nasal type is a rare, clinically aggressive disease with a 1year survival rate of $40 \%$ [1]. It is more common in males in their 50s, and in Asia and Latin America, it represents $7-10 \%$ of all non-Hodgkin lymphomas [1-3]. ENKL of the nasal type is often localized in the upper aerodigestive tract, including the nasal cavity, nasopharynx, paranasal sinuses, tonsils, hypopharynx and larynx [4, 5]. However, it can present with extranasal involvement, and the most common sites of extranasal involvement or metastatic disease include the skin, testis and gastrointestinal tract. The most common clinical features of ENKL include nasal obstruction or nasal bleeding due to a mass lesion $[4,5]$.

In a report of 73 patients with ENKL of the nasal type from the MD Anderson cancer center, $60 \%$ of the patients had localized disease (stage I or II), and 10 patients had extranasal disease with skin involvement in 3 of them [6]. Skin manifestations of NKL can be quite varied and are therefore often initially missed by providers, which leads to incorrect staging and treatment. The most common skin manifestation of ENKL is a well-defined, erythematous and violaceous nodule that most often appears on the extremities and trunk [1, 7]. While this is the most common presentation, other manifestations, such as papules, cysts, patches and plaques, have been reported. Due to this varied presentation, any suspicious or worrisome lesion in a patient with NKL should be biopsied before staging and treatment initiation [7]. Whether the lesions are solitary or generalized seems to have no effect on prognosis [3]. 
Al Akhrass et al.: Extranodal Natural Killer/T-Cell Lymphoma of the Nasal Type with Skin Metastases

The histology of such skin lesions shows angiocentricity and angiodestruction with a polymorphic lymphoid infiltrate [1-3]. The immunophenotype is CD3 epsilon+, CD56+, CD2+, CD3- and often CD20- $[1-3,7]$. There is no clonal rearrangement of the T-cell receptor, and cytotoxic molecules containing granzyme B, TIA- 1 and perforin are often found $[1,2$, 7]. EBV is usually associated with the lesions and can be measured by quantitative PCR for a marker of tumor burden [2]. It has been noted that cases of NKL in the United States tend to have a decreased incidence of EBV, which is thought to be secondary to the lower rates of infectivity that are seen in the US compared to Asia and Latin America [3].

Diagnosis and treatment are highly dependent on staging. Complete staging includes flexible nasal panendoscopy with biopsies to rule out nasal involvement, bone marrow biopsy, chest, abdominal and pelvic computed tomography scans, and biopsies of any suspicious cutaneous lesions $[2,3,7]$. Radiotherapy can be beneficial in patients with stage I or II disease that is mainly limited to the nasal cavity, but beyond that radiotherapy has limited efficacy [2]. The primary treatment of ENKL is systemic chemotherapy. The CHOP regimen is commonly used, but newer regimens such as SMILE (steroids, methotrexate, ifosfamida, pegaspargase and etoposide) that contain L-asparaginase are showing promise in clinical trials. These newer regimens appear to be more specific to NKL due to the fact that these tumor cells lack L-asparagine synthetase and thus are more susceptible to L-asparaginasebased regimens $[1,2]$. Other treatment options with variable efficacy are allogenic hematopoietic stem cell transplantation with the benefit of the graft-versus-lymphoma effect, antiviral therapy for patients with a high EBV load, and monoclonal antibody therapy that is still in the research phase [1,2].

In conclusion, ENKL of the nasal type is a rare and highly aggressive type of lymphoma. Although the disease is mainly limited to the nasal cavity, cutaneous manifestations can represent skin metastases and should be evaluated adequately with a biopsy of any suspicious lesion since it will change the staging and affect the treatment and prognosis.

\section{Statement of Ethics}

The authors have no ethical conflicts to disclose.

\section{Disclosure Statement}

The authors have no conflicts of interest to declare.

\section{References}

1 Gill H, Liang R, Tse E: Extranodal natural-killer/T-cell lymphoma, nasal type: review article. Adv Hematol 2010;2010:e1-e5.

2 Berti E, Recalcati S, Girgenti V, et al: Cutaneous extranodal NK/T-cell lymphoma: a clinicopathologic study of 15 patients with array-based comparative genomic hybridization. Blood 2010;116:165-170.

3 Mraz-Gernhard S, Natkunam Y, Hoppe R, et al: Natural killer/natural killer-like T-cell lymphoma, CD56+, presenting in the Skin: an increasingly recognized entity with an aggressive course. J Clin Oncol 2001;19:2179-2188.

-4 Pincus L, Zehnder J, Neuhaus I, et al: Presentation of extranodal natural killer T-cell lymphoma, nasal type, with poorly circumscribed erythematous patches. J Clin Oncol 2010;28:e94-e95.

5 Shaoying L, Xiaoli F, Ting L, et al: Extranodal NK/T-cell lymphoma nasal type. A report of 73 cases at MD Anderson Cancer Center. Am J Surg Pathol 2013;37:14-23. 


\section{Case Reports in Oncology}

\begin{tabular}{l|l}
\hline Case Rep Oncol 2016;9:1-5 \\
\hline DOI: 10.1159/000443161 & $\begin{array}{l}\text { @ 2016 The Author(s). Published by S. Karger AG, Basel } \\
\text { www.karger.com/cro }\end{array}$ \\
\hline
\end{tabular}

Al Akhrass et al.: Extranodal Natural Killer/T-Cell Lymphoma of the Nasal Type with Skin Metastases

6 Leblond V, Sutton L, Dorent R, et al: Lymphoprolferative disorders after organ transplantation: a report of 24 cases observed in a single center. J Clin Oncol 1995;13:961-968.

7 Leblond V, Dhedin N, Mamzer Bruneel MF, et al: Identification of prognostic factors in 61 patients with posttransplantation lymphoproliferative disorders. J Clin Oncol 2001;19:772-778.

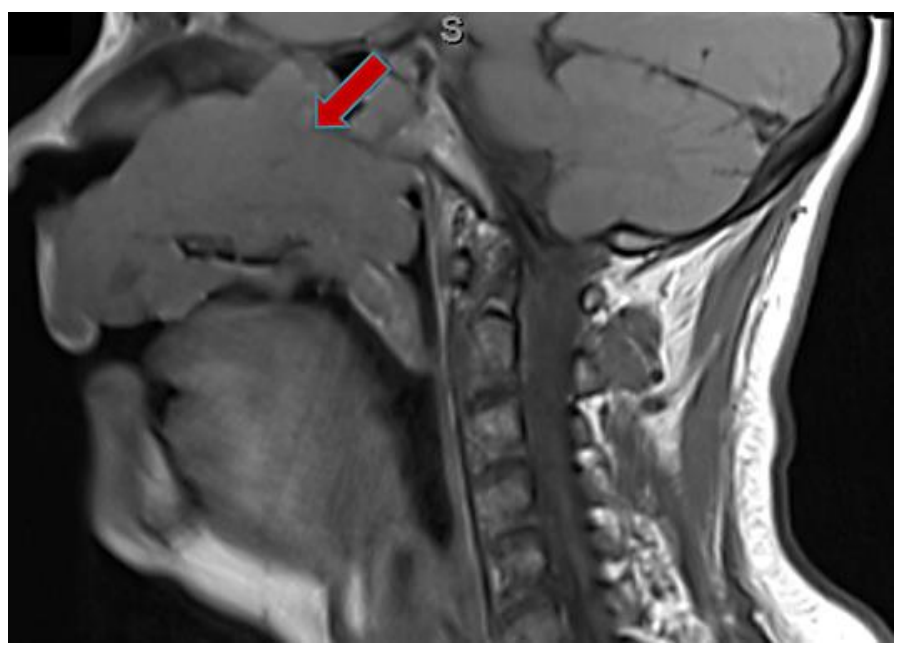

Fig. 1. MRI of the face with contrast medium revealed the presence of an $8.3 \times 4.8-\mathrm{cm}$ heterogeneous mass lesion involving both nasal airways with obstruction and destruction of the nasal septum. There is extension into the underlying hard palate and into the left ethmoid, sphenoid, left maxillary sinuses, soft palate and uvula.

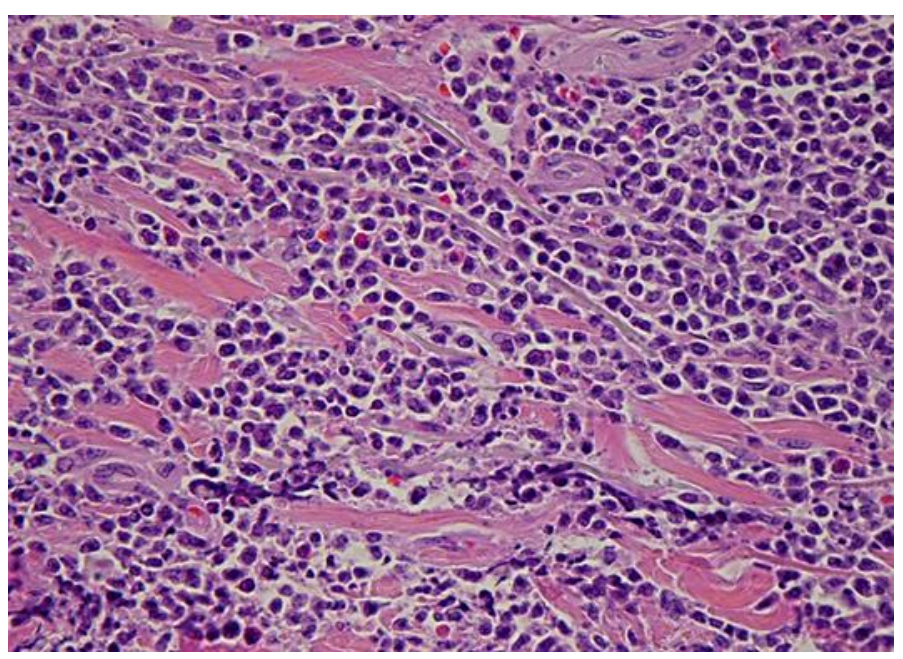

Fig. 2. Sections demonstrate a dense, diffuse, pandermal proliferation of atypical lymphoid cells with eosinophilic cytoplasm, hyperchromatic nuclei, irregular nuclear membranes and inconspicuous nucleoli. Extensive apoptosis, prominent angiocentricity, focal epidermotropism and dermal necrosis are noted. The tumor cells were strongly immunoreactive for TIA-1, granzyme, CD8, CD2, CD56 and cytoplasmic CD3. A few cells were immunoreactive for CD5 and CD4. EBER in situ hybridization was positive in the majority of the neoplastic cells. The cells were negative for CD57. The findings are consistent with ENKL of the nasal type. 
Al Akhrass et al.: Extranodal Natural Killer/T-Cell Lymphoma of the Nasal Type with Skin Metastases

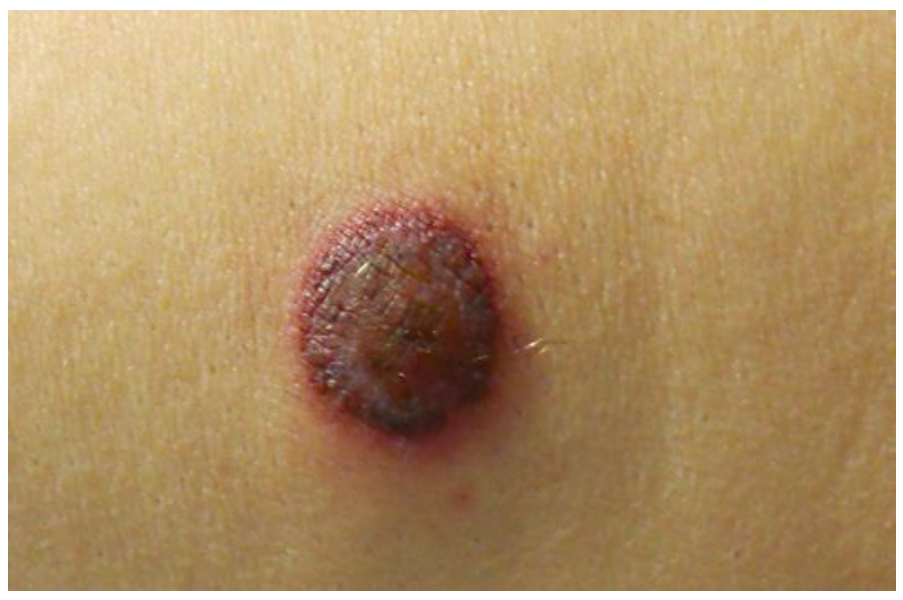

Fig. 3 . A $3 \times 3-\mathrm{cm}$, erythematous, violaceous, well-circumscribed nodular lesion on the left thigh. 